\title{
Diversité Biologique Et Structure De La Faune Ichtyologique De l'Aire Marine Protégée De Joal-Fadiouth Au Sénégal
}

\author{
Mamadou Ndiaye, \\ Direction des Aires Marines Communautaires Protégées, Dakar, Sénégal \\ Massal Fall, \\ UFR Gestion des Ressources Halieutiques, Pêche et aquaculture, Université \\ du Sine Saloum El Hadj Ibrahima Niasse (USSEIN), Fatick, Sénégal \\ Malick Diouf, \\ Institut Universitaire de Pêche et d'Aquaculture, \\ Université Cheikh Anta Diop de Dakar, Sénégal
}

Doi:10.19044/esj.2020.v16n12p11～URL:http://dx.doi.org/10.19044/esj.2020.v16n12p11

\section{Résumé}

Cette étude vise à mieux faire connaître le fonctionnement écologique de l'aire marine protégée (AMP) de Joal-Fadiouth via l'inventaire de sa composition spécifique et la répartition spatiale de sa faune ichtyologique. A cet effet, un suivi bioécologique a été mené durant les 4 saisons hydrologiques de 2015 à 2017, au niveau de 6 stations caractéristiques des habitats de l'AMP, avec les mêmes engins et équipe de pêcheurs. Les analyses statistiques ont été effectuées à l'aide du logiciel XLSTAT 2017. Au bilan, 120 espèces de poissons appartenant à 86 genres et 48 familles ont été identifiées. Les Carangidae sont les plus nombreux avec 13 espèces. Elles sont suivies par les Sparidae (08), les Haemulidae et Mugilidae (07 chacune), les Solidae (06), les Sciaenidae et les Tetraodontidae (05), les Polynemidae et Serranidae (4 chacune), et 6 et 11 autres familles avec respectivement, 3 et 2 espèces. Les 23 autres familles affichent chacune une seule espèce. Seules 2 espèces parmi les 70 jusque-là identifiées par des études antérieures n'ont pas été retrouvées par cette étude qui a permis de revoir cette liste avec 57 nouvelles espèces. Cette richesse spécifique est inégalement répartie dans l'AMP. La station " Kher-ciment » présente la richesse spécifique la plus importante avec 75 espèces, soit $59 \%$ de l'ichtyofaune de l'AMP de Joal-Fadiouth. Elle est suivie de celles du Quai de pêche (70 espèces, $55 \%$ ), du Bolong Mbissel et Ngousse Diohé (65 espèces pour chacune, soit 51,18 \%), du Bolong Mama Nguedj (64 espèces, $50 \%$ ) et de la Zone « tortues » (48 espèces, $38 \%$ ). Chacune de ces zones présente une richesse spécifique supérieure au maximum de 52 espèces jusque-là rencontré dans une zone et largement au-delà des 18 espèces d'une 
station dominante dans l'AMP de Cayar. Avec ces résultats, cette confirme l'importance de ces zones pour le fonctionnement écologique de l'AMP.

Mots clés: Sénégal, Aire Marine Protégée, Joal-Fadiouth, Diversité Biologique, Faune Ichtyologique

\title{
Biological Diversity and Structure of the Ichthyological Fauna of the Marine Protected Area of Joal-Fadiouth (Senegal)
}

\author{
Mamadou Ndiaye, \\ Direction des Aires Marines Communautaires Protégées, Dakar, Sénégal \\ Massal Fall, \\ UFR Gestion des Ressources Halieutiques, Pêche et aquaculture, Université \\ du Sine Saloum El Hadj Ibrahima Niasse (USSEIN), Fatick, Sénégal
}

\section{Malick Diouf,}

Institut Universitaire de Pêche et d'Aquaculture, Université Cheikh Anta Diop de Dakar, Sénégal

\begin{abstract}
This study aims to raise awareness of the ecological functioning of the Joal-Fadiouth marine protected area (MPA) through the inventory of its specific composition and the spatial distribution of its ichthyological fauna. To this end, bioecological monitoring was carried out during the 4 hydrological seasons from 2015 to 2017, at 6 stations characteristic of MPA habitats, with the same gear and team of fishermen. The statistical analyzes were performed using the XLSTAT 2017 software. In the balance, 120 species of fishes belonging to 86 genera and 48 families have been identified. The Carangidae are the most numerous with 13 species. They are followed by Sparidae (08), Haemulidae and Mugilidae (07 each), Solidae (06), Sciaenidae and Tetraodontidae (05), Polynemidae and Serranidae (4 each), and 6 and 11 other families with 3 and 2 species respectively. The other 23 families each display a single species. Only 2 of the 70 species so far identified by previous studies were not found by this study, which made it possible to review this list with 57 new species. This specific richness is unevenly distributed in the MPA. The "Kher-cement" station has the highest specific richness with 75 species, or $59 \%$ of the ichthyofauna of the Joal-Fadiouth MPA. It is followed
\end{abstract}


by those of the fishing wharf (70 species, 55\%), Bolong Mbissel and Ngousse Diohe (65 species for each, or 51.18\%), Bolong Mama Nguedj (64 species, $50 \%$ ) and "Turtles" area (48 species, 38\%). Each of these zones has a specific richness greater than the maximum of 52 species hitherto encounted in a station of MPA, and far beyond the 18 species identified in a dominant station in the Cayar MPA. With these results, this study confirm the importance of these zones for the ecological functioning of the MPA.

Keywords: Marine Protected Area, Senegal, Joal-Fadiouth, Biological Diversity, Ichtyological Fauna

\section{Introduction}

Les zones côtières et marines abritent des habitats-clés pour les cycles biologiques de nombreuses espèces marines (Man-Waï, 1985 ; García-Rubies \& Macpherson, 1995 ; Harmelin-Vivien et al., 1995 ; Macpherson et al., 1997 ; Planes et al., 1999 ; Planes et al., 2000). Mais au cours de ces deux dernières décennies, l'intensification croissante de la pression sur les ressources halieutiques et l'accélération alarmante des processus de dégradation des habitats marins font planer le risque majeur de régression d'espèces (Bretagnolle et al., 2000 ; Rogers, 2001 et Lalèyè, 1995). Au regard de ces problèmes globaux, qui ne permettent plus d'assurer la pérennité des pêcheries (Murray et al., 1999 ; Pauly et al., 2002), la protection des habitats et de la ressource est devenue une préoccupation majeure. La nécessité d'adopter des mesures de gestion permettant de réduire la surexploitation des ressources et la dégradation des habitats marins est devenue, dès lors, un souci mondialement partagé. Conformément aux recommandations issues du Sommet de Johannesburg sur le Développement durable en 2002, le Sénégal a poursuivi ses efforts, par la création en 2004 de 5 AMP par le décret $n^{\circ} 1408$ du 18/11/2004. Il s'agit des AMP de Saint-Louis, Kayar, Joal-Fadiouth, Bamboung et Abéné. Depuis 2012, la Direction des Aires Marines Communautaires Protégées (DAMCP) s'est attelée à accompagner les initiatives des communautés locales notamment, au niveau du delta du Saloum et en Casamance. Cela a abouti à la création, toujours par décret, de 4 nouvelles AMP (Sangomar, Gandoule, Niamone-Kalounayes et KassaBalantacounda), toutes dotées de plans d'aménagement et de gestion (Fig.1). Ceci a nettement amélioré le taux de couverture nationale en AMP qui est passé de $1,2 \%$ en 2012 à $2,44 \%$ en 2018, en perspective de l'atteinte de l'objectif 14.5 d'Aïchi, à savoir « D'ici à 2020, préserver au moins $10 \%$ des zones marines et côtières, conformément au droit national et international ».

L'objectif global poursuivi, à travers la création de tous ces espaces protégés, est la préservation de la productivité et de la biodiversité des zones marines et côtières. Avec les multiples effets écologiques sur les communautés 
benthiques et démersales, pour les augmentations d'abondance, de biomasse et/ou de taille moyenne (Bell, 1983 ; Alcala \& Russ, 1990 ; García-Rubies \& Zabala, 1990 ; Roberts \& Polunin, 1993 ; Attwood et al., 1997 ; Agardy, 1998 ; Allison et al., 1998 ; Caddy, 2000 ; Francour et al., 2001 ; Lubchenco et al., 2003), la préservation et la durabilité de la ressource (White et al., 1990), l'Aire Marine Protégée de Joal-Fadiouth (AMPJF) constitue, à l'instar des autres, un important outil de gestion locale des pêches. La diversité des communautés biologiques, que les AMP en général peuvent assurer, joue un rôle déterminant dans le fonctionnement des écosystèmes (Weis et al. 2000 ; Roy et al. 2001 ; Kinzing et al., 2002 ; Sherman et Anderson 2002). L'outil de gestion des pêches qu'est, potentiellement, l'approche écosystémique (Ecosystemic Approach to Fisheries/EAF, en anglais), pour être efficace, nécessite une bonne connaissance des espèces et des relations qui les lient à leur milieu (Sadio, 2015). Or, paradoxalement au Sénégal, il n'y a que très peu d'inventaires exhaustifs de la faune ichtyologique des aires de conservation.

Les informations disponibles sur l'ichtyofaune sont très fragmentaires et restent limitées en milieux estuariens et en zone de mangrove (Ecoutin et al., 2012 ; Simier et al., 2004 ; Diouf, 1996), à la limite aux zones de pêche démersale côtières (Thiam et al, 2013). Les plus importants travaux de recherche sur les AMP ont beaucoup plus porté sur des aspects d'efficacité de la gestion (Sène, 2014 et Sadio, 2015). L'AMPJF ne fait pas exception à la règle, les connaissances sur son fonctionnement écologique étant très limitées ou presque inexistantes. Il en résulte une impérieuse nécessité aujourd'hui de faire le point sur sa richesse ichtyologique, en attendant l'extension de l'étude aux autres représentants de sa faune (crustacés, mollusques, etc.).

L'AMP de Joal-Fadiouth est caractérisée par une diversité d'habits naturels comprenant des zones de mangroves, d'herbiers marins, de bolongs et de zones pierreuses ; toutes choses qui favorisent une richesse ichtyologique très importante non maîtrisée par les gestionnaires de cette aire. Dans le cadre de la stratégie nationale pour les AMP du Sénégal, la DAMCP en partenariat avec l'institut Universitaire de Pêche et d'Aquaculture (IUPA), le Centre de Recherches Océanographiques de Dakar-Thiaroye (CRODT) et la Direction des Pêches Maritimes (DPM) a entrepris un important programme de suivi bioécologique des AMP, dont celle de Joal-Fadiouth. Cette démarche stratégique, approfondie avec les travaux d'une thèse en cours, a permis de procéder à un inventaire systématique de la faune ichtyologique de ce site durant les années 2015, 2016 et 2017. 


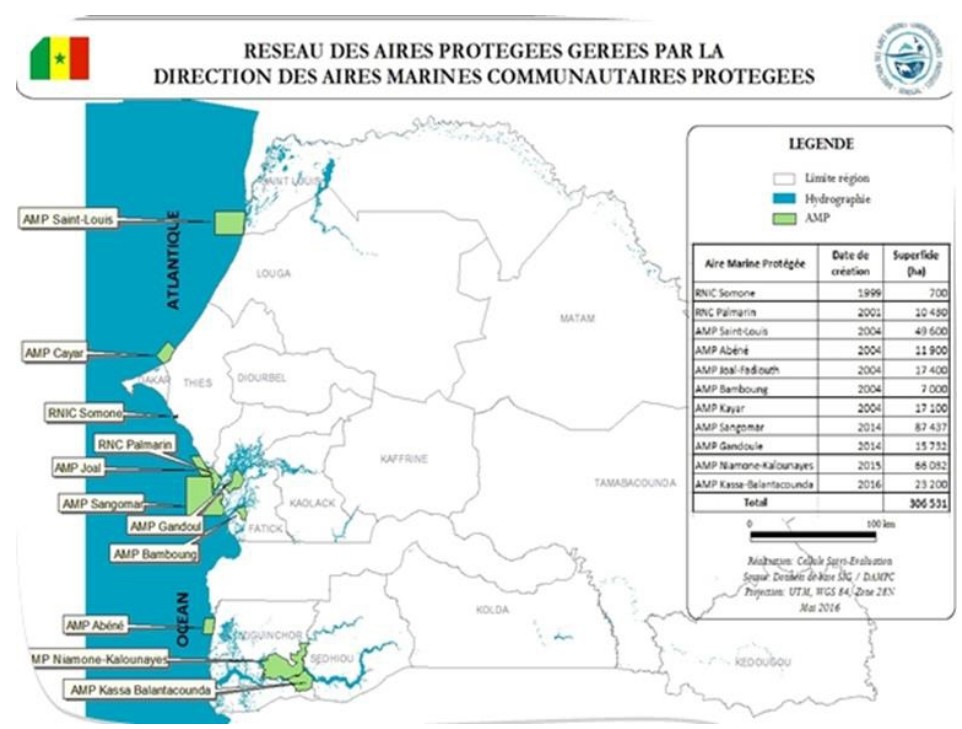

Figure 1 : Réseau des aires marines protégées du Sénégal

Les principaux matériels et méthodes mis en œuvre ainsi que les résultats obtenus durant ces périodes sont exposés ci-après.

\section{Matériels et méthodes}

\section{Présentation de la zone d'étude}

L'AMPJF (Fig. 2) est située dans la région de Thiès, département de Mbour, commune de Joal-Fadiouth. Elle s'étend sur $174 \mathrm{~km} 2$ dans sa partie marine et comprend, dans sa partie continentale, un bras de mer et un important réseau de mangroves. Avec sa position géographique sur la petite côte du Sénégal, l'AMPJF subit de plein fouet l'influence des facteurs physiques qui caractérisent cette partie du plateau continental sénégalais. Sa structure bathymétrique présente des profondeurs relativement faibles (Sarré et al, 2009), de l'ordre de 3 à $7 \mathrm{~m}$, favorables au bon développement de la flore marine (Carte 2). Ses caractéristiques hydrologiques restent dominées par trois grandes masses d'eaux dont la répartition varie suivant les saisons (Rossignol, 1973 in Fréon, 1986) (i) les eaux froides et salées, canariennes $\left(\mathrm{T}<24^{\circ} \mathrm{C}\right.$; S $>35 \%$ ), dont les manifestations sont beaucoup plus importantes dans la zone de janvier à février (ii) les eaux tropicales, chaudes et salées $\left(\mathrm{T}>24^{\circ} \mathrm{C} ; \mathrm{S}>\right.$ $35 \%$ ), qui envahissent la zone à partir du mois de mai jusqu'en octobre (iii) les eaux guinéennes, chaudes et dessalées $\left(\mathrm{T}>24^{\circ} \mathrm{C} ; \mathrm{S}<35 \%\right)$, venant du sud et dont la dessalure est liée essentiellement aux apports des grands fleuves de la région guinéenne. Deux systèmes de grands courants balayent la zone (Fréon, 1986) : le courant des Canaries en saison froide et le contre-courant équatorial qui apporte les eaux chaudes et salées en saison chaude. L'hydro climat est assez contrasté, avec deux grandes saisons maritimes, la saison 
froide et la saison chaude qui peuvent donner des saisons intermédiaires, le réchauffement et le refroidissement dont les caractéristiques majeures sont exposées dans le Tableau 1.

\begin{tabular}{|c|c|}
\hline Saisons & Principales caractéristiques \\
\hline $\begin{array}{l}\text { Saison froide } \\
\text { (décembre à mai) }\end{array}$ & $\begin{array}{ll}\text { - } & \text { manifestation des alizés (continental/harmattan et } \\
\text { maritime/lié à l'anticyclone des Açores) à dominante } \\
\text { nord-nord-ouest } \\
\text { - } \quad \text { période d'upwelling par excellence (eaux canariennes) }\end{array}$ \\
\hline $\begin{array}{l}\text { Transition saison froide- } \\
\text { chaude (mai à juin) }\end{array}$ & $\begin{array}{ll}\text { - } & \text { transition saison froide/saison chaude } \\
\text { - } & \text { début d'apparition des eaux tropicales chaudes }\end{array}$ \\
\hline $\begin{array}{l}\text { Saison chaude } \\
\text { (juin à novembre) }\end{array}$ & $\begin{array}{ll}\text { - } & \text { atténuation des alizés et de la remontée d'eau froide } \\
\text { - } & \text { manifestation de la mousson, apparition des pluies } \\
\text { - } & \text { eaux tropicales et guinéennes } \\
\end{array}$ \\
\hline $\begin{array}{l}\text { Transition saison } \\
\text { chaude-froide } \\
\text { (refroidissement) } \\
\text { (novembre à décembre) }\end{array}$ & $\begin{array}{ll}\text { - } & \text { transition saison chaude/saison froide } \\
\text { - } & \text { retrait des eaux tropicales chaudes vers le sud } \\
\text { - } & \text { début d'apparition des eaux canariennes froides }\end{array}$ \\
\hline
\end{tabular}

Tableau 1 : Saisons hydrologiques et caractéristiques Sources: Rossignol et Aboussouan (1965) et Rébert (1979) in Fall (2009)

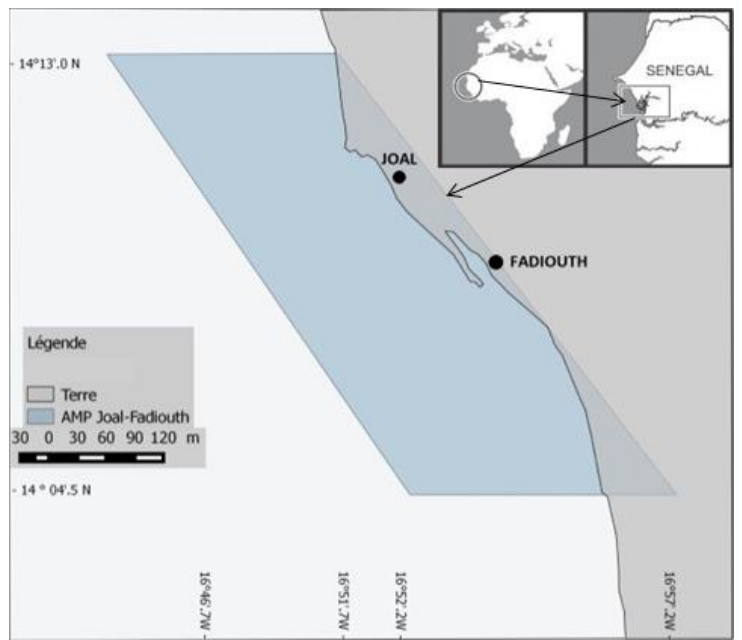

Figure 2. Localisation et situation géographique de l'Aire Marine Protégée de JoalFadiouth.

\section{Collecte des données}

Il est communément admis que l'établissement d'un protocole d'échantillonnage est une étape décisive qui va conditionner la qualité de l'interprétation des résultats (Baran, 1995). En effet, Livingston (1987) a montré que la méconnaissance des échelles de variabilité spatiales et temporelles d'un système étudié pouvait conduire à des interprétations erronées lorsque l'échelle de variation propre au phénomène étudié était inférieure à l'échelle d'observation retenue. Afin d'avoir une bonne représentativité de la diversité ichtyologique de l'AMPJF, 6 stations de pêche 
ont été choisies au hasard au niveau des différents habitats naturels (Fig. 3) pour y effectuer des échantillonnages biologiques durant les 4 saisons maritimes (Tableau 1) des années 2015 à 2017.

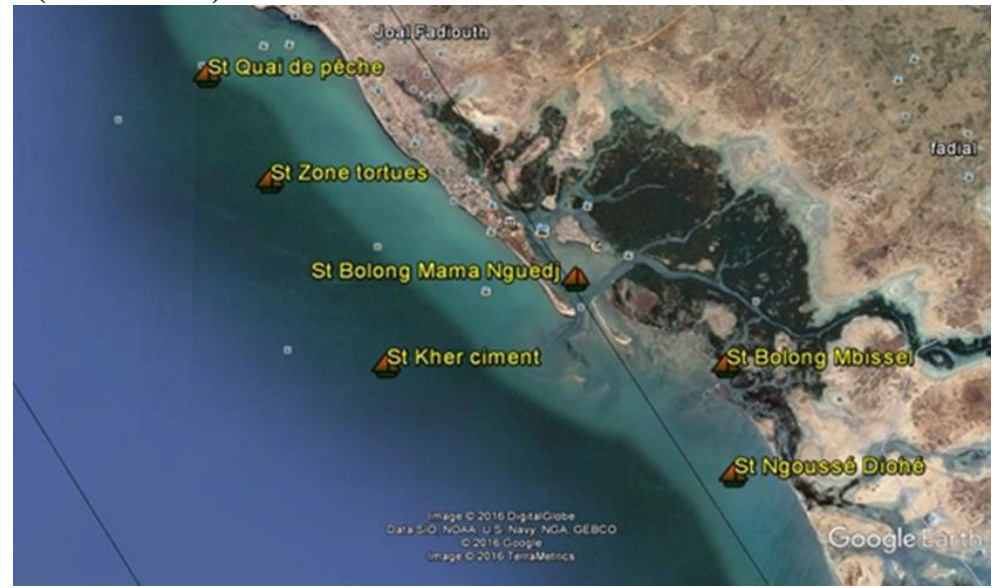

Figure 3 : Localisation des six stations d'échantillonnage biologiques dans l'AMPF

Pour ce qui est de la technique d'échantillonnage, la perception du " peuplement réel » souhaitée est totalement dépendante de la méthode de pêche employée, du fait de l'accès possible ou non de l'engin à certaines zones de l'AMPJF, de la sélectivité et de l'efficience de cet engin (Baran, 1995). Ainsi, 4 types d'engin de pêche : une senne tournante (ST), une senne de plage (SP), un filet maillant encerclant (FME) et une palangre de mailles $12 \mathrm{~mm}$ étirée ont été utilisées de manière à obtenir l'échantillonnage le plus complet possible de l'ichtyofaune de l'AMPJF. Ces engins ont des effets complémentaires car à même de pêcher des représentants des ressources pélagiques et/ou benthodémersales.

L'identification des espèces a été faite grâce à la « Faune des poissons d'eaux douces et saumâtres de l'Afrique de l'Ouest » (Lévêque et al, 1992), ou, si les espèces ne figuraient pas dans ce premier ouvrage, les fiches FAO d'identification des espèces de l'Atlantique (1981), ou suivant Blache et al. (1970).

\section{Traitement des données}

Les résultats ont été dans un premier temps présentés sous la forme d'une liste d'espèces de poissons identifiées dans l'ensemble de l'AMPJF en 2015, 2016 et 2017. Pour mieux synthétiser l'information et avoir une idée plus globale des peuplements de poissons, les échantillons des différents prélèvements ont été regroupés par station pour une étude spatiale et ceux des différentes stations par saison pour une analyse temporelle de l'ichtyo faune. Les informations recueillies au niveau des 6 stations de la pêche expérimentale ont fait l'objet d'analyses mathématiques et statistiques au moyen du logiciel 
XLSTAT ${ }^{\circledR}$ (2017). Pour l'étude quantitative, nous avons utilisé deux indices biologiques synthétiques calculés à partir des effectifs, à savoir :

a) l'indice de diversité spécifique $\left(H^{\prime}\right)$ calculée selon la formule de

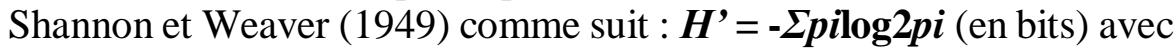
$p i=\mathrm{ni} / \mathrm{N}, \mathrm{N}$ étant la somme totale des effectifs (individus) obtenus pour toutes les espèces ; ni, le nombre d'individus par espèce ; $p i$ l'abondance relative de l'espèce i dans l'échantillon

b) l'indice de régularité de Piélou (1969) ou équitabilité (E), défini comme étant le rapport de la diversité réelle à la diversité maximale, est calculé. Il s'exprime par la formule $\mathbf{E}=\boldsymbol{H}^{\prime} / \log 2 \mathrm{~S}$, avec E compris entre 0 et $1 . S$ est la richesse spécifique.

L'absence de normalité dans la distribution des données et le nombre de séries à analyser (6 stations et 12 mois de pêche expérimentale) ont conduit à utiliser des tests non paramétriques pour comparer les captures entre les différentes stations ou entre les échantillonnages mensuels d'une même station. Des analyses statistiques ont été également effectuées. Après le calcul des indices écologiques, et pour vérifier si les résultats obtenus ont un vrai sens statistique, le test non paramétrique de Kurskal-Wallis a été appliqué pour comparer plusieurs séries de valeurs entre elles. Les différences en composition spécifique ont été évaluées par des dendrogrammes de similarité obtenus après le calcul de l'indice de dissimilarité de Bray-Curtis avec le logiciel Past $3{ }^{\circledR}$. Une analyse qualitative fondée sur la présence ou l'absence des espèces dans les différents échantillons a été faite à l'aide de l'indice de Jaccard (1908) qui mesure la similarité entre deux communautés et exprimé comme suit : J_d=N_ab/((N_a+N_b-N_ab ) ) Avec Na : nombre d'espèces dans la station $\mathrm{a} ; \mathrm{Nb}$ : nombre d'espèces dans la station $\mathrm{b}$ et $\mathrm{Nab}:$ nombre d'espèces communes dans les stations a et $b$.

Afin de faciliter l'identification des patrons de distribution des dix espèces dominantes au niveau des 6 stations et durant les 4 saisons de la période 2015-2017 et de confirmer les résultats obtenus avec les précédentes analyses univariées, nous avons utilisé une analyse en composantes principales (ACP). Cette analyse multivariée, basée sur la variance spécifique des variables, permet d'extraire un minimum de facteurs qui expliquent la plus grande partie possible de la variance spécifique. Elle nous a permis de condenser l'information contenue à l'intérieur du grand nombre de variables (10 espèces) en un ensemble restreint de nouvelles dimensions composites tout en assurant une perte minimale d'informations. Dans ce cas précis d'une décision confirmatoire, et pour être certain de bien choisir le bon nombre de facteurs à extraire, le critère du coude Cattell a été utilisé. Le principe consiste, là, à regarder le graphique des valeurs propres et examiner où se situe la rupture du coude dite de. 
Dans un premier temps, les données ont été transformées en leur logarithme naturel pour assurer une distribution normale et standardisée en vue de leur centrage (moyenne ramenée à 0 ) réduction (écart type $=1$ ). Ensuite, elles ont été arrangées en forme de matrice comme suit : les lignes représentant les stations d'échantillonnage et les colonnes, les variables à étudier (c.-à-d. les espèces et les saisons).

\section{Résultats}

\section{Inventaire de la faune ichtyologique}

L'inventaire de la faune ichtyologique de l'AMPJF de 2015 à 2017 a permis d'identifier les différentes espèces et leur aire de répartition.

$\mathrm{Au}$ total, 120 espèces ont été recensées, entre les 6 stations inventoriées. Elles se répartissent en 86 genres appartenant à 48 familles. L'ordre des familles est celui adopté par Lévêque et al. (1990-1992).

Les Carangidae sont les plus nombreux avec 13 espèces. Elles sont suivies par les Sparidae avec 08 espèces. Les Haemulidae et Mugilidae viennent après avec chacune 07 espèces, puis les Soleidae avec 06 espèces. Ensuite les Sciaenidae et Tetraodontidae avec 05 espèces. Les Polynemidae et Serranidae sont représentées chacune par 04 espèces. Six (06) et dix (10) familles sont représentées respectivement par 3 et 2 espèces. Toutes les 23 familles restantes ne comportent qu'une seule espèce.

Ces espèces appartiennent à cinq catégories écologiques. Cent vingt (120) d'entre elles, issues de 44 Familles, sont typiquement d'essence marine ou estuarienne. Dans ces catégories écologiques, les familles les plus représentées sont les Carangidae, Sparidae, Mugilidae et Soleidae avec respectivement 13,8 et 7 espèces.

En ce qui concerne les abondances spécifiques de la faune ichtyologique de l'AMPJF, les 10 espèces dominantes sont Carlarius parkii $(43,7 \%)$,

Eucinostomus melanopterus $(15,1 \%)$, Ethmalosa fimbriata $(10,9 \%)$, Ilisha africana $(7,5 \%)$, Sarotherodon melanoteron heudelotti $(3,6 \%)$, Pomadasys incisus (2,6\%), Diplodus bellottii $(2,4 \%)$, Mugil bananensis (1,3\%), Galeoides decadactylus (1,2\%) et Liza dumerili (1,0\%) (Fig.4).

Ces espèces appartiennent à plusieurs familles dont les 6 dominantes sont réparties de manière différente d'une station à une autre. Les Carangidae et les Sparidae sont plus nombreux au niveau de la station Quai de pêche, avec respectivement 8 et 7 espèces. Les Haemulidae et Clupeidae dominent dans la station Ngoussé diohé avec respectivement 6 et 4 espèces. Par contre, les deux autres familles présentent chacune le même nombre d'espèces au niveau de deux stations : il s'agit des Mugilidae (7 espèces au niveau des stations Bolong Mama Nguedj et Bolong Mbissel) et des Tetraodontidae (4 espèces dans chacune des stations Mbolong Mbissel et Kher ciment) (Fig.5). 


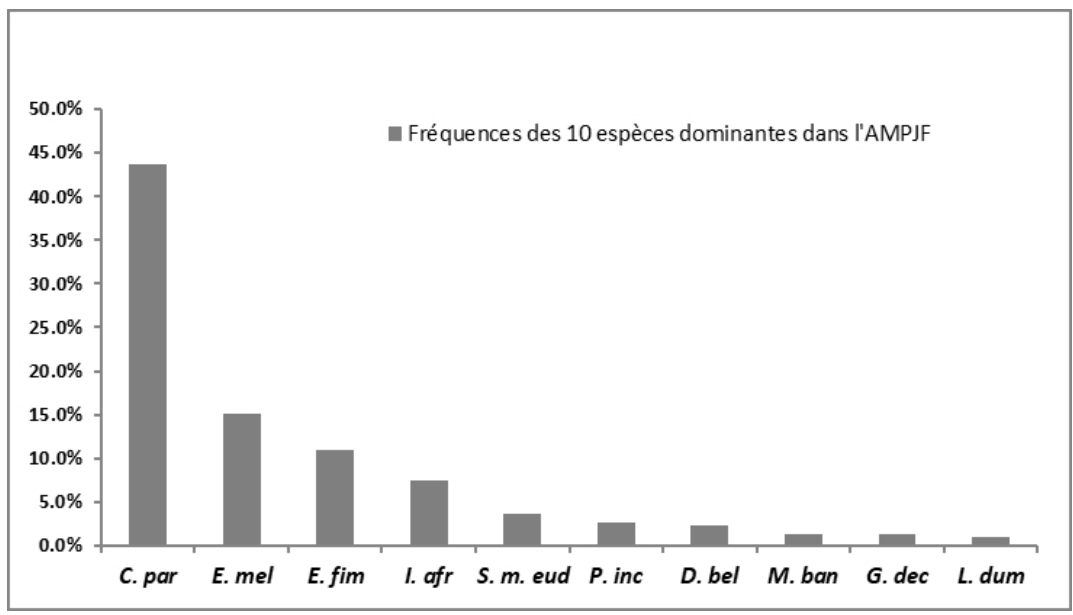

Figure 4 : Fréquences des dix espèces dominantes du peuplement de poissons de l'AMPJF :

Carla parkii ; C par : Ethmalosa fimbriata ; E fim : Eucinostomus melanopterus ; E mel : Ilishia africana ; I afr : Sarotherodon melanoteron heudelotti ; $S \mathrm{~m}$ eud : Diplodus bellottii ; $D$ bel : Mugil bananensis ; M ban : Galeoides decadactylus ; G dec : Liza duerili ; $L d u$.

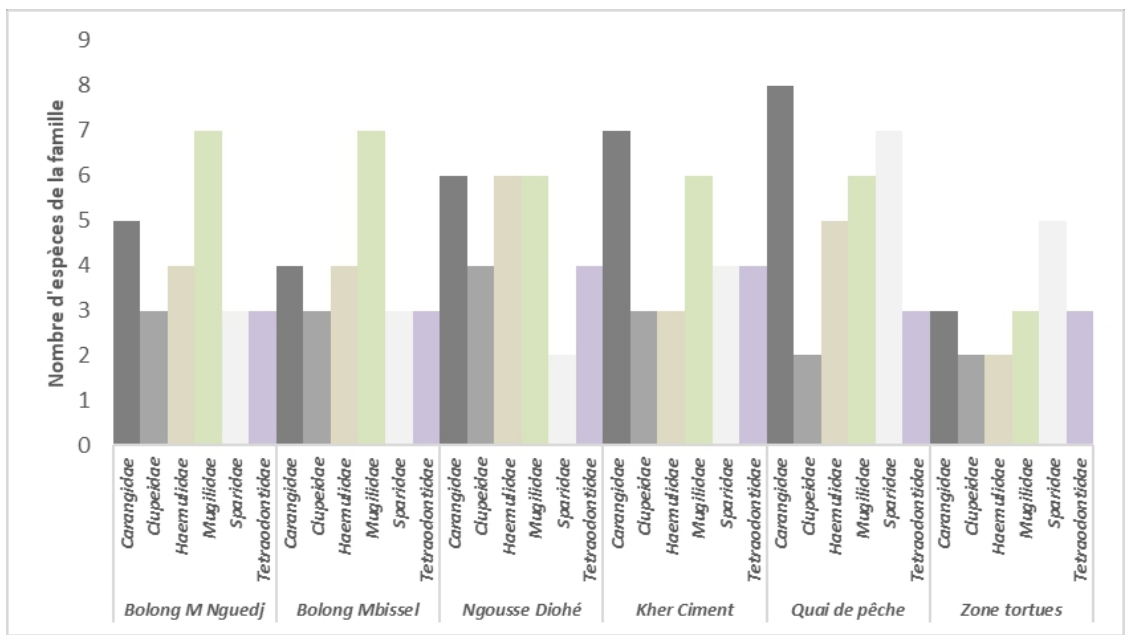

Figure 5 : Nombre d'espèces des six familles dominantes dans les six stations de l'AMPJF

L'étude de la répartition temporelle de la faune ichtyologique de l'AMPJF au cours de la période 2015-2017, montre que la diversité spécifique varie d'une saison à une autre.

Ainsi, le plus grand nombre d'espèces (99 espèces) a été identifié en saison froide, suivi des transitions entre les saisons froide et chaude (67 espèces) et chaude et froide (65 espèces), ainsi que durant la saison chaude avec un recensement de 70 espèces au niveau des 6 stations. 


\section{Répartition spatiale de la faune ichtyologique}

La répartition spatiale des espèces varie d'une station à l'autre. Sur la base des résultats d'observation des captures de la pêche expérimentale de la période 2015-2017, la station Kher ciment présente la richesse spécifique la plus importante (75 espèces, 59,1\% de l'ensemble de l'ichtyofaune), suivie par celles du Quai de pêche (70 espèces, 55,12\%), Bolong Mbissel et Ngousse Diohé avec chacune 65 espèces $(51,18 \%$ ), Bolong Mama Nguedj (64 espèces, $50,39 \%$ ) et Zone tortues (48 espèces, 37,79 \%) (Fig. 6). D'une saison à une autre, cette différence en termes de richesse spécifique entre les stations n'est pas significative $(\mathrm{n}=24 ; \mathrm{ddl}=18 ; \mathrm{H}=16,48 ; \mathrm{P}>0,05)$.

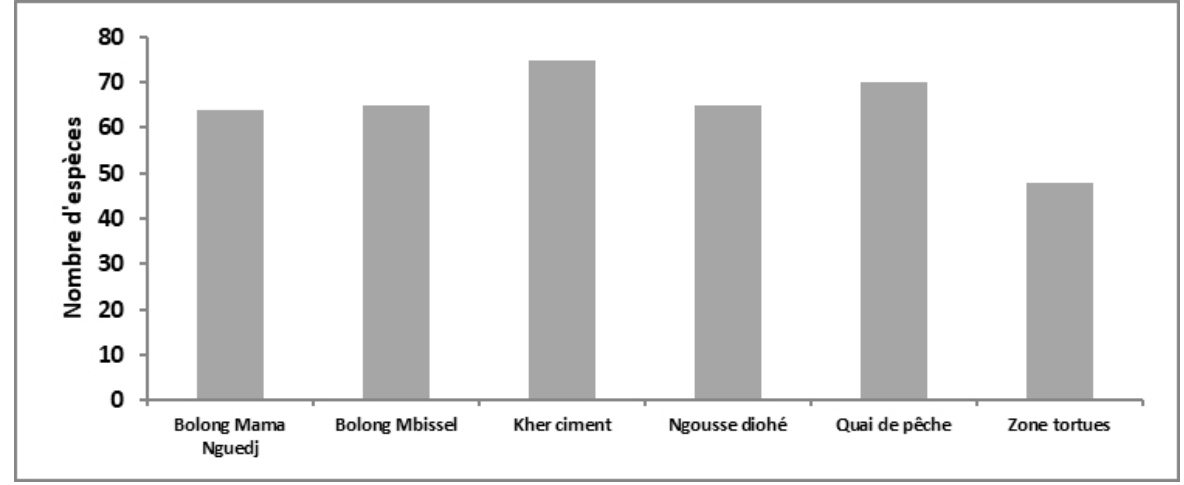

Figure 6 : Richesse spécifique au niveau des 6 stations d'échantillonnage

Contrairement à la richesse spécifique, des disparités assez importantes sont notées dans les variations de la fréquence des 10 espèces dominantes. Quatre (4) espèces, Ethmalosa fimbriata, Eucinostomus melanopterus, Carlarius parkii et Ilisha africana, représentent plus de la moitié de la population au niveau de chacune des stations, à l'exception de celle de zone tortues où les fréquences cumulées des 10 espèces précitées font près de $18 \%$.Ainsi, les stations Bolong Mbissel, Ngoussé Diohé, Quai de pêche et Zone tortues sont dominées respectivement par Eucinostomus melanopterus (41\%), Carlarius parkii $(84,7 \%)$, Ilisha africana $(62,4 \%)$ et Eucinostomus melanopterus (10,8\%). Par contre, les stations Bolong Mama Nguedj et Kher ciment sont dominées respectivement par deux espèces : Eucinostomus melanopterus $(32,6 \%$ et $22,2 \%$ ) et Ethmalosa fimbriata $(35,4 \%$ et $22,2 \%$ ) (Fig.7). Ainsi, E. melanopterus est le taxon-phare dans 3 des 6 stations de pêche. 


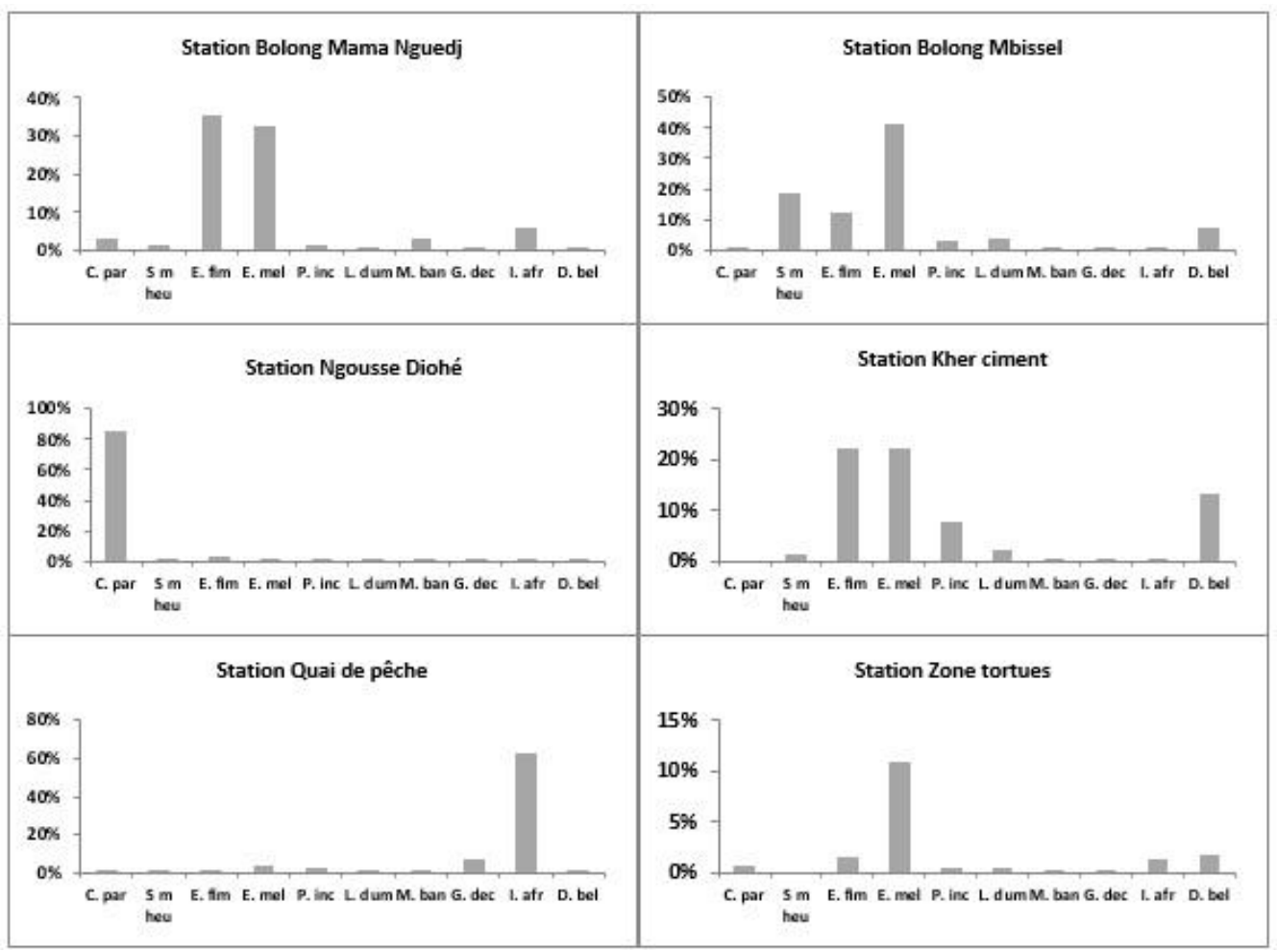

Figure 7 : Fréquences des 10 espèces dominantes dans les 6 stations d'échantillonnage de l'AMPJF

Une confrontation des fréquences spécifiques des 6 stations révèle plus de divergence que de similitudes. La plus remarquable similarité (55\%) est notée entre les stations Bolong Mam Nguedj (BMN) et Bolong Mbissel (BOM) comme l'indique le dendrogramme de similarité (Fig.8). Les deux espèces responsables de cette situation sont Ethmalosa fimbriata et Eucinostomus melanopterus. Pour toutes les autres combinaisons possibles, les similarités inter-stations sont inférieures à $30 \%$. De ce constat, certes d'énormes écarts sont observables sur les fréquences spécifiques d'une station à l'autre, mais globalement plus de la moitié des espèces sont présentes dans toutes les stations. Ce qui constitue un autre facteur pouvant alléger la différence, une hypothèse confirmée par le test de Kruskal-Wallis $(\mathrm{p}=0,1833>0,05)$. 


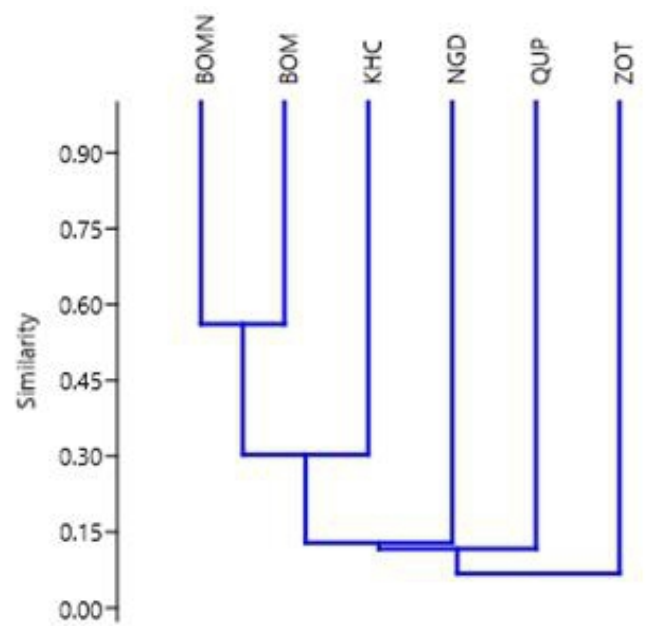

Figure 8 : Dendrogramme de similarité des fréquences des dix espèces dominantes de l'AMPF dans les stations

Des variations spatiales sont également notées sur les indices de diversité. L'indice de diversité de Shannon-Weaver (Sh) varie en moyenne entre 2,4 et 1,2, avec des valeurs extrêmes atteignant 2,77 (valeur maximale) à la station Quai de pêche et 0,21 (minimum) à la station Ngoussé Diohé (Fig. 9a).

Mis à part cette dernière station où des accroissements de l'indice de Shannon-Weaver ont été notés au cours des 3 premières saisons, l'indice de Shannon évolue en dents de scie au niveau de toutes les autres stations (Fig. 10). Croissance-décroissance au niveau des stations zone tortues et Quai de pêche, ensuite décroissance-croissance au niveau des stations Bolong Mama Nguedj; Bolong Mbissel et Kher ciment.

L'ANOVA de Kruskal-Wallis $(\mathrm{N}=24 ; \mathrm{ddl}=5 ; \mathrm{H}=5,995 ; \mathrm{p}=0,306$ $(>0,05))$ et le test de la médiale (Chi-deux $=6 ; \mathrm{ddl}=5 ; \mathrm{p}=0,3)$ réalisés pour évaluer l'intensité de cette différence notée sur l'évolution de l'indice de Shannon-Weaver, montrent qu'il n y'a pas de différence significative à ce niveau.

Par ailleurs, l'équitabilité de Piélou quant à elle, évolue en moyenne entre 0,44 et 0,75 avec des extrêmes de 0,96 au niveau de la station Zone tortues (valeur maximale) et 0,06 à Ngoussé Diohé (valeur minimale) (Fig. 9b). 

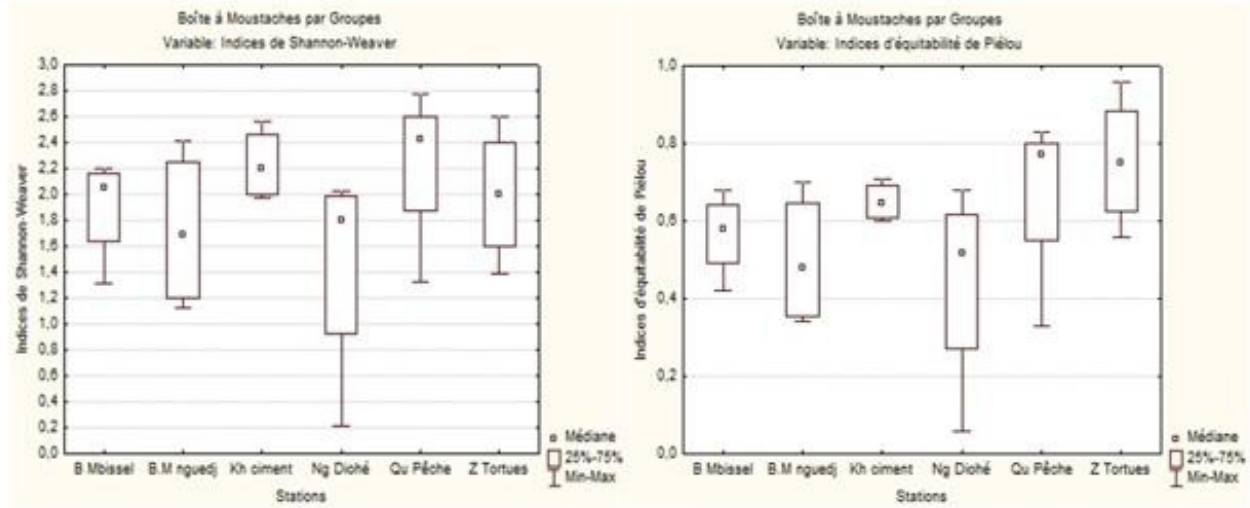

Figure 9 : Valeurs moyennes par stations des indices écologiques : a) Indice de Shannon-Weaver et b) Indice d'équitabilité de Piélou

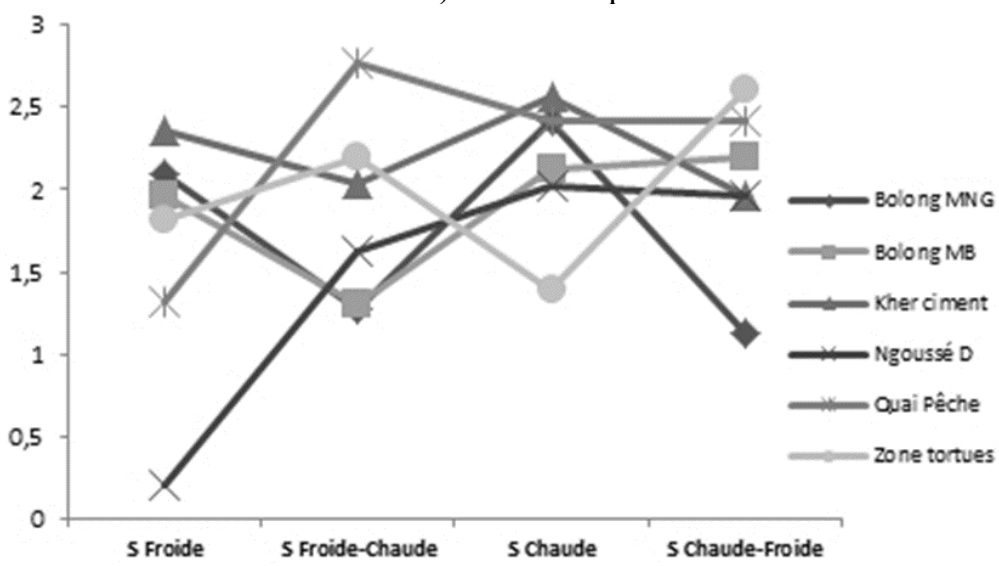

Figure 10 : Variations de l'indice de Shannon-Weaver par stations au cours des quatre saisons

Le traitement statistique de ces différentes valeurs de l'équitabilité de Piélou à l'aide d'un test de Kruskal-Wallis montre une différence non significative entre les six stations $(\mathrm{N}=24 ; \mathrm{ddl}=5 ; \mathrm{H}=7,56 ; \mathrm{P}>0,05)$.

L'indice d'équitabilité de Piélou présente des valeurs supérieures à 0,75 uniquement au niveau des stations Quai de pêche ( 0,83 en saison froidechaude ; 0,77 en saison chaude et en saison chaude-froide) et Zone tortues ( 0,96 en saison froide-chaude et 0,81 en saison chaude-froide). Ces valeurs prouvent une répartition équitable du peuplement de poissons pour les espèces présentes dans ces deux stations au cours des saisons citées.

Les valeurs de l'indice de Jaccard obtenues entre les six stations pour les quatre saisons au cours des trois dernières années restent assez moyennes. Elles varient entre $59,26 \%$ et $31,11 \%$ avec une moyenne de $46,52 \%$. Les similarités les plus élevées sont observées entre les stations Bolong Mama Nguedj et Bolong Mbissel (59,26\%), entre les stations Ngoussé Diohé et 
Bolong Maman Nguedj (55,42\%) et entre Kher ciment et Bolong Missel $(52,17 \%)$. Par contre, les valeurs les plus faibles se situent entre les stations Quai de pêche et Zone tortues (31,11), Ngoussé Diohé et Zone tortues $(39,51 \%)$, Kher ciment et Zone tortues $(41,38 \%)$. Il en ressort que la station Zone tortues est celle qui présente moins de similarité avec les cinq autres stations.

En considérant la répartition spatiale des dix espèces dominantes du peuplement de poissons de l'AMPJF, les degrés de similarité entre stations sont presque identiques. Ainsi, l'analyse en composantes principales (ACP) effectuée à partir du tableau abondances numériques a permis d'opposer sur l'axe 1 (94\% de l'information) la station Ngoussé diohé aux cinq autres stations (Fig. 11). On peut même dire que cet axe 1 est caraactéristique de Ngoussé diohé. Les grandes similarités observées avec les stations Bolong Mama nguedj et Bolong Mbissel sont bien exprimées par cette ACP. L'axe 2 (4\% de l'information) oppose les bolongs de Mama Nguedj et Mbissel d'une part aux 3 stations restantes de Kher ciment, Zone tortues et Quai de pêche, d'autre part.

En termes de variables explicatifs, deux groupes d'espèces qui ont un rôle dominant dans la formation des deux axes se dégagent. Le premier groupe est composé de Carlarius parkii, Mugil bananensis et Pomadasys incisus qui ont le plus contribué à la formation de l'axe 1. Ces espèces présentent une plus grande affinité pour la station Ngoussé diohé. Quant au deuxième groupe d'espèces contributives de l'axe 2, il y'a Eucinostomus melanopterus, Ethmalosa fimbriata, Sarotherodon melanoteron heudeloti et Diplodus bellottii. Ces espèces sont beaucoup plus affiliées aux stations Bolong maman nguedj et Bolong mbissel.

Variables et Individus (axes F1 et F2: $98 \%$ )

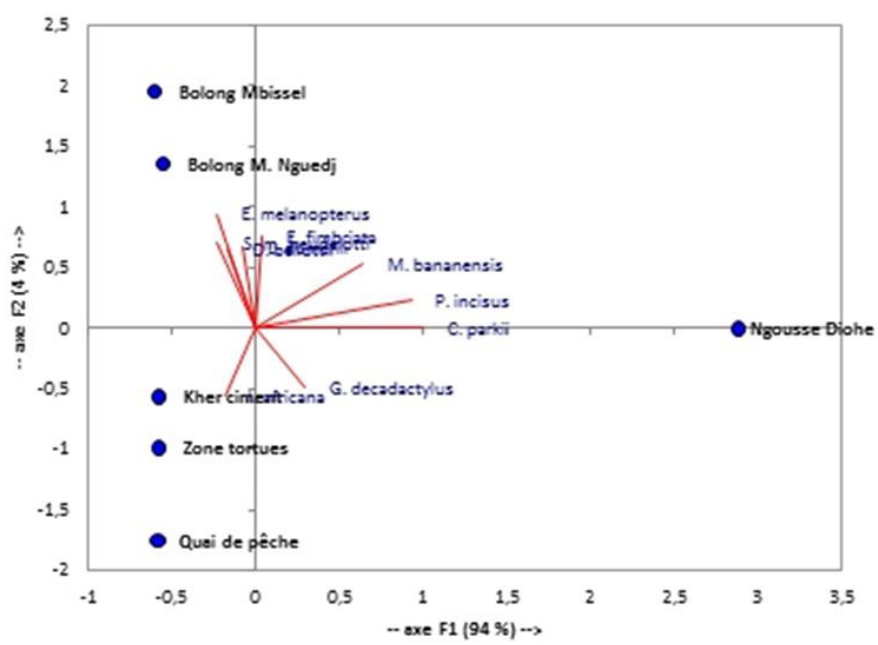

Figure 11 : Analyse en composantes principales représentant les stations et les espèces sur le plan composé par les deux principaux axes 1 et 2 . 


\section{Variations temporelles de la faune ichtyologique de l'AMPJF}

Les variations temporelles de l'ichtyofaune ont été étudiées au niveau des six stations au cours de 12 saisons hydrologiques de 2015 à 2017. A ce niveau, les variations saisonnières ont été étudiées séparément.

Les résultats obtenus à cet effet, montrent que la composition spécifique du peuplement de poissons varie au sein d'une même station, d'une saison à une autre. La figure 10 présente l'évolution de la richesse ichtyologique au niveau de chacune des 6 stations en fonction des saisons hydrologiques de la période 2015-2017. En dehors des transitions saison chaude-saison froide des deux premières années (SCHF_2015 et SCHF_2016), la station Zone tortues présente les plus petites richesses spécifiques durant les autres saisons. Pour les autres stations, les variations de la richesse spécifiques sont assez différentes au point qu'il n'existe pas de similitudes particulières pour ces dernières au cours des différentes saisons hydrologiques. L'analyse de la variance (test d'ANOVA) effectuée sur les données de richesses spécifiques des six stations au cours des trois années, présente des différences significatives $(p<0,0001)$ sur l'ensemble des six stations.

D'une saison à une autre, la richesse spécifique de l'AMP varie de 28 à 56 espèces (Fig. 12a). La plus grande richesse spécifique a été rencontrée en saison froide 2016 (SF_2016) et le minimum durant la transition saison froidechaude (TFCH_2015). De manière générale, au cours de chaque année, le plus grand nombre d'espèces est enregistré en période froide. L'évolution du nombre de familles des espèces durant les différentes saisons hydrologiques suit aussi le même gradient de variation (Fig. 12b). Le plus grand nombre de familles est rencontré durant la saison froide de la période 2015-2017.

Le test non paramétrique de Kruskal-wallis effectué sur la composition spécifique au cours des 6 saisons, hydrologique pour la période 2015-2017, montre l'existence d'une différence significative entre les saisons $(\mathrm{p}=0,01686<0,05)$.
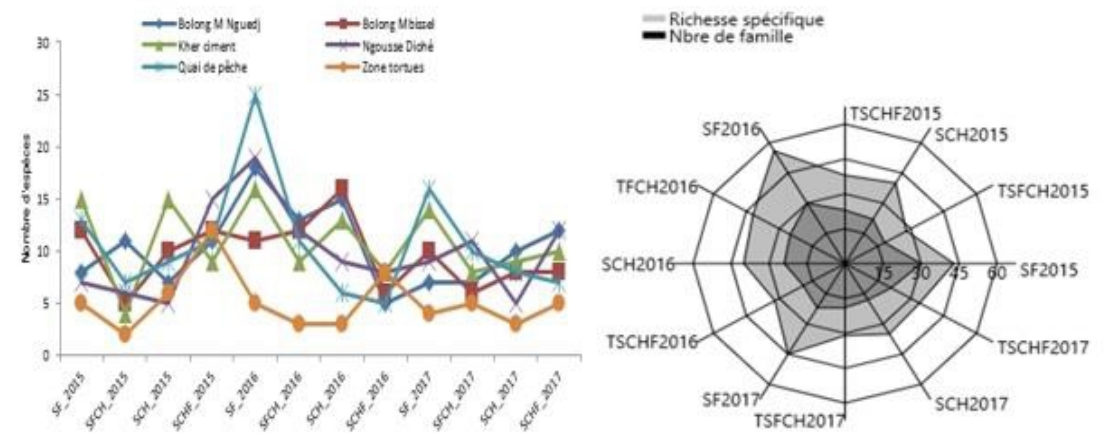

Figure 12 : Variation au cours des quatre saisons hydrologiques de 2015_2017 : a) de la richesse spécifique de et b) du nombre de familles des espèces de l'AMPJF 
Les deux analyses en composantes principales effectuées à partir des tableaux des abondances numériques au cours de la première année (2015) et de la deuxième année (2016) de suivi, présentent pratiquement les mêmes résultats. L'ACP des données de la première année (Fig. 13a) présente un axe 1 constitué à $75 \%$ par la saison froide (SF) et un axe 2 qui oppose la saison de transition froide-chaude (SFCH avec 60,57\% de contribution) à la saison chaude (SCH avec 32,57\%) et la saison transition chaude-froide (SCHF avec $6,61 \%$ de contribution). Les espèces responsables de ces variations sont Carlarius parkii, Ethmalosa fimbriata et Eucinostomus melanopterus pour l'axe 1 et l'axe 2 oppose l'espèce Liza dumerili aux espèces Pomadasys incisus, Diplodus bellottii, Mugil bananensis et Galeoides decadactylus.

De la même manière, l'ACP des données de la deuxième année (Fig.13b), qui présente les quatre saisons suivant les mêmes dispositions, avec quelques différences près pour les contributions à la formation des axes 1 et 2 . A ce niveau, l'axe 1 est principalement constitué par les espèces Galeoides decadactylus, Ilisha africana, Carlarius parkii et Ethmalosa fimbriata. Contrairement à l'axe 2 qui oppose légèrement les espèces Sarotherodon melanotheron heudelotti et Eucinostomus melanopterus, aux espèces Mugil bananensis, Diplodus bellottii, Pomadasis incisus et Liza dumerili. Pour chacune de ces deux ACP, le plan formé par les deux premiers axes explique respectivement $95 \%$ et $100 \%$ de la variance totale obtenue.

En ce qui concerne la répartition des dix espèces dominantes au cours des quatre saisons hydrologiques de 2017, l'ACP effectuée à partir du tableau des abondances, montre que les plus importantes abondances de poissons sont observées respectivement durant les saisons transition chaude-froide, froide et froide-chaude (Fig. 13c).

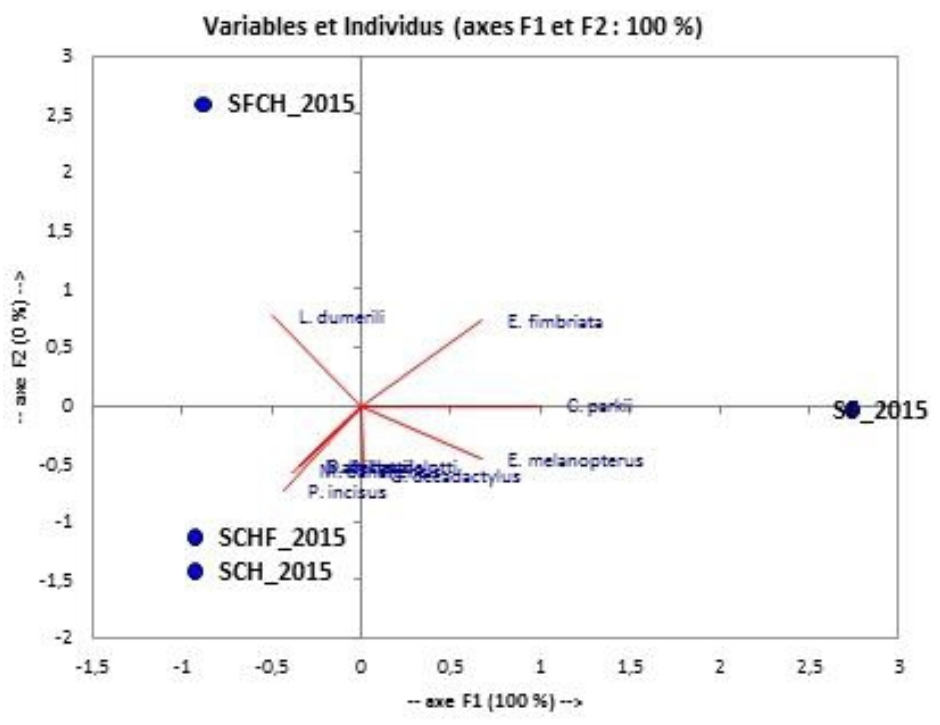



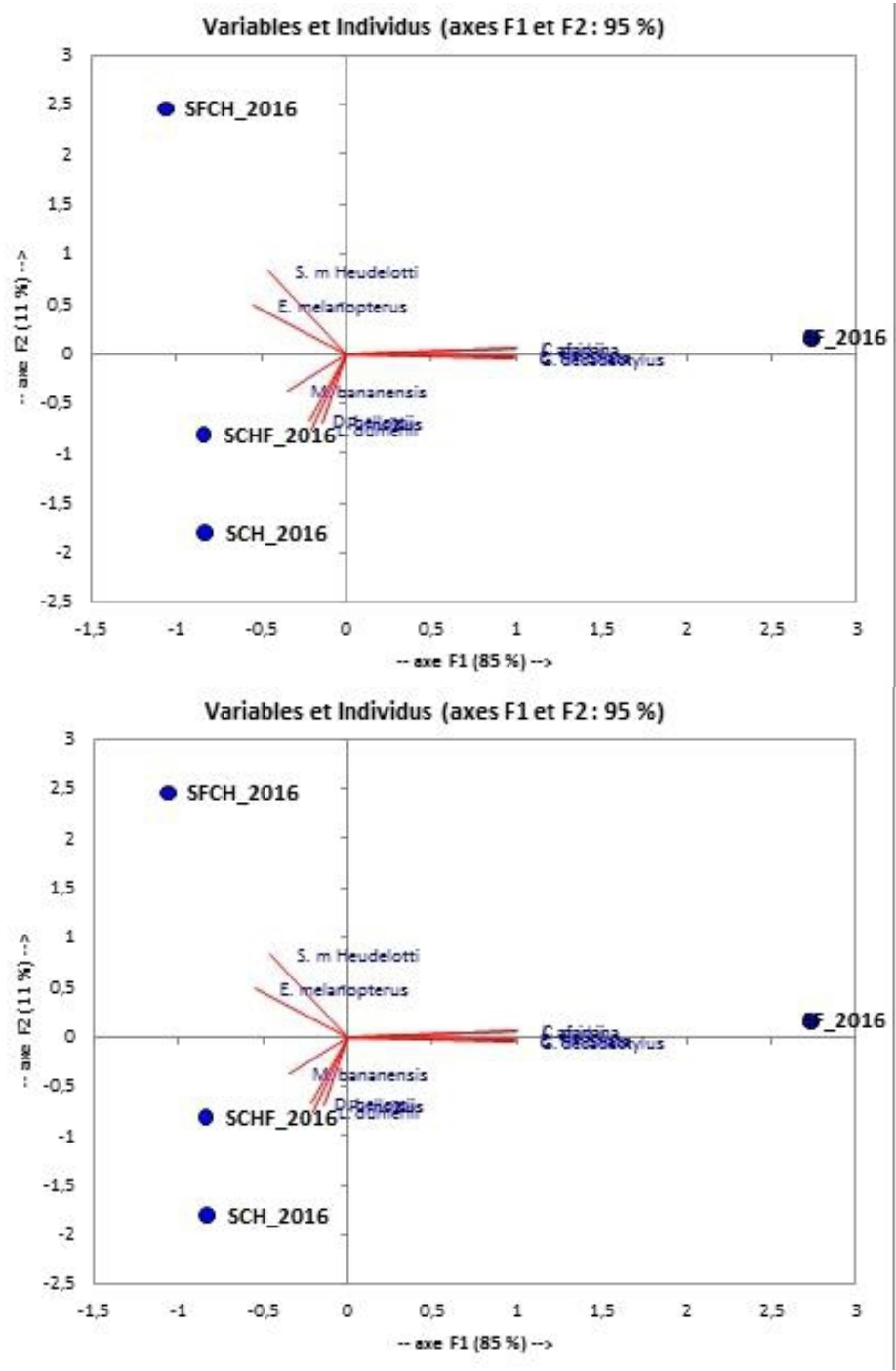

Figure 13 : Projection des saisons années d'échantillonnage dans le plan comportant les deux premiers axes principaux : a) première année d'échantillonnage (2015), b) deuxième année d'échantillonnage (2016) et c) troisième année d'échantillonnage (2017).

En regardant de près les fréquences des dix espèces dominantes du peuplement de poissons de l'AMPJF, on constate des similarités entre certaines saisons hydrologiques (Fig.14). Les plus importantes similarités apparaissent entre les deux transitions saison chaude-saison froide de 2015 et celle de 2016 (TSCHF2015 et TSCHF2015) qui se distinguent par la même identité remarquable qui est l'absence de 5 espèces parmi les 10 dominantes durant ces 2 saisons. La seconde importante similarité se situe entre la saison froide et la transition saison froide-chaude de 2017 (SF2017 et TSFCH2017). Le caractère commun entre ces 2 saisons est l'existence de 6 espèces parmi les 
10, qui se présentent avec pratiquement les mêmes proportions. Il s'agit des espèces Carlarius parkii, Ethmalosa fimbriata, Eucinostomus melanopterus, Liza dumerili, Diplodus bellottii et Galeodes decadactylus.

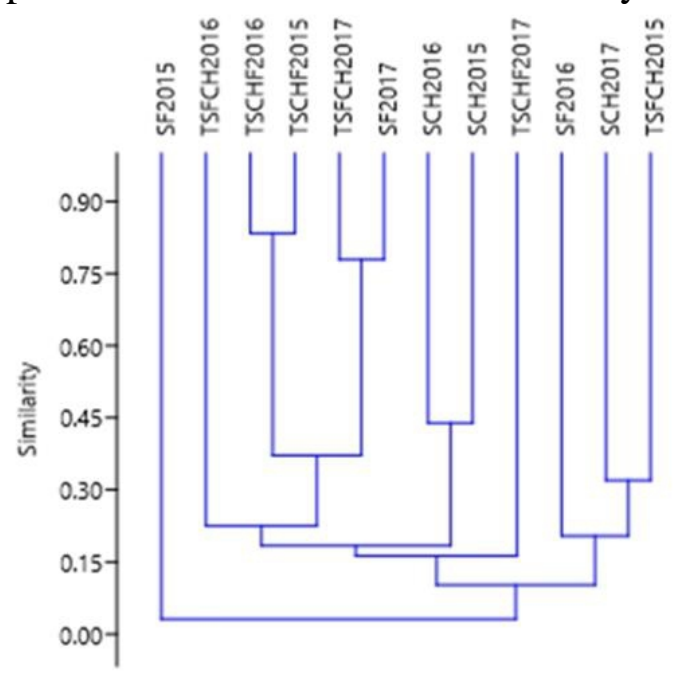

Figure 14 : Dendrogramme des similarités de la composition spécifique de l'AMPJF au cours des différentes saisons hydrologiques.

\section{Discussion}

$\mathrm{Au}$ regard des opérations régulières d'inventaires réalisées avec différents engins de pêche durant les quatre saisons hydrologiques et au niveau des 6 zones caractéristiques des habitats de l'AMP de Joal-Fadiouth, il est probable qu'une partie appréciable des espèces présentes ont été identifiées. En effet, ces opérations de prospection ont duré trois ans avec $63 \%$ des espèces capturées et identifiées lors de la première année, $32 \%$ au cours de la deuxième année et seulement $5 \%$ à la dernière année. Cette régression remarquable du pourcentage d'apparition de nouvelles espèces au cours des deux dernières années nous conforte dans l'idée d'un inventaire ichthyologique relativement exhaustif.

Sans trop exagérer, encore moins sous-estimer les résultats des études antérieures, on peut sans doute dire qu'il y'a une meilleure évaluation de la richesse spécifique du peuplement de poissons de l'AMP de Joal-Fadiouth au cours de ces trois dernières années. La comparaison des résultats de notre étude avec celles menées par Sarre et al. (2009) et DAMCP (2014) révèle une augmentation du nombre d'espèces de poissons passé de 56 espèces en 2009, 79 espèces en 2014 et 120 espèces en 2017.

De 2005 à nos jours, c'est le même mode de gouvernance de l'AMPJF qui est en vigueur, avec l'interdiction des autres formes de pêche (à part les éperviers, la pêche sportive et les filets de maille $100 \mathrm{~mm}$ ) et de toute autre activité pouvant perturber le bon fonctionnement écologique de l'AMP. 
Partant de ce fait, ces différences de résultats pourraient être liées aux méthodologies utilisées et plus particulièrement aux types d'engins de pêche utilisés et aux périodes d'échantillonnage. Un postulat qui semble se confirmer avec les travaux de Sarre et al (2009) qui avaient utilisé uniquement une senne de plage au niveau de trois stations et seulement en saison froide (sur les quatre saisons).

En comparant les résultats de la DAMCP (2014) avec ceux de Sarr et al. (2014), on pourrait penser que la saisonnalité de certaines espèces de poissons a beaucoup influé sur cette différence de nombre (79 contre 70). Contrairement à Sarr et al. (2014), les travaux de DAMCP (2014) ont été étendus à l'ensemble des quatre saisons hydrologiques en 2014, avec le même nombre de stations de pêche.

Les zones abritant les herbiers marins, les récifs artificiels et les estuaires de mangrove appelées respectivement par cette étude, Zone tortues, Kher ciment et les deux Bolongs n'ont pas été prospectées par ces auteurs. Ces zones présentent des caractéristiques bioécologiques particulières, offrant des niches écologiques spécifiques à certaines catégories d'espèces de poissons qu'on ne peut retrouver nulle part ailleurs dans l'AMP. Ce qui fait qu'un inventaire exhaustif de la faune ichtyologique doit nécessairement tenir compte de ces habitas particuliers.

$\mathrm{Au}$ sein du même continuum écologique de la réserve de biosphère transfrontière du delta du Saloum, la richesse spécifique de 1'AMP de JoalFadiouth, comparée à celle des espaces halieutiques du Sine Saloum comme l'estuaire du Sine Saloum (111 espèces ; Diouf, 1996), l'AMP de Bamboung (67 espèces ; Sadio, 2005) et le complexe AMP Bamboung, chenal du Diomboss et le bolong de Sangako (77 espèces ; Ecoutin et al. (2012) est plus importante.

Un des facteurs qui explique la richesse en espèces de l'AMP de JoalFadiouth est la diversité des habitats (herbiers marins, bras de mer, multitudes de bolongs, présence de mangroves, aménagements de récifs, diversité des sédiments de fond, variations spatio-temporelles des facteurs physicochimiques...).

Cette dominance de la richesse spécifique de l'AMP de Joal-Fadiouth serait corroborée par les résultats du suivi bioécologique de la DAMCP (2014) au niveau de neuf sites du réseau d'AMP du Sénégal présentant l'AMP de Joal-Fadiouth au sommet avec 86 espèces, devant l'AMP d'Abéné (61 espèces), AMP Bamboung (43 espèces), AMP Cayar (66 espèces), AMP Gandoul (63 espèces), AMP Joal-Fadiouth (86 espèces), AMP Saint-Louis (31 espèces), AMP Sangomar (74 espèces), RNC Palmarin (27 espèces) et RNIC Somone (23 espèces)

Parmi ce cortège de 120 espèces inventoriées dans l'AMP au cours des pêches expérimentales des trois années de suivi bioécologique, la majeure 
partie, $80,31 \%(\mathrm{n}=102)$ contribue chacune pour moins de $0,19 \%$ (soit moins de 100 individus) à l'effectif total échantillonné (47 029 individus). Ces espèces ont été absentes ou en très petits nombres dans beaucoup de stations. Les plus rares d'entre elles sont les poissons de grande taille appartenant principalement aux familles des Carangidae (Caranx chrysos, Campogramma glaycos, Trachinotus maxillosus, Scorpaena laevis, Caranx hyppos....) et des Sparidae (Dentex gibbosus, Diplodus sargus, Diplodus vulgarus...).

Du point de vue de la répartition spatiale de l'ichtyofaune, même si la différence de richesse spécifique entre stations n'est pas significative, les stations Kher ciment et Quai de pêche ont les richesses spécifiques les plus élevées. Ces deux stations sont situées respectivement dans la zone de transition entre la mer et l'espace estuarien pour la station Kher ciment et le milieu lagunaire pour la station Quai de pêche.

Les importantes richesses spécifiques notées au niveau des bolongs Mama nguedj et Mbissel peuvent s'expliquer par le fait que ces écosystèmes aquatiques constituent des zones fonctionnelles halieutiques pour beaucoup d'espèces de poissons. De nombreuses études menées à travers le monde, dans ces types d'écosystèmes de mangroves, ont conduit au paradigme de l'utilisation de ces milieux par un grand nombre d'espèces marines comme nourricerie (Warburton, 1978 ; Beckley, 1985 ; Cyrus and Blaber, 1987 et Baran, 1995).

Ces bolongs, avec les importantes formations de mangroves qui les entourent, représentent une voie directe de transfert d'énergie vers les milieux adjacents et offrent les conditions nécessaires à la reproduction, la ponte, la croissance jusqu'à la maturité et l'alimentation tout au long de la vie pour plusieurs espèces halieutiques (Magnuson-Stevens Fishery Act, 2007). Ces deux bolongs offrent aux juvéniles et aux larves une nourriture abondante, des biotopes d'eaux calmes et une protection contre la prédation du fait de la turbidité et de la présence d'abris (racines de palétuviers, herbiers, zones peu profondes...) (Warburton, 1978 ; Potter et al., 1990 ; Blaber et al., 1985; Wootton, 1992 ; Cyrus and Blaber, 1987 ; Lenanton and Hodgkin, 1985). Ce sont des zones où s'accumulent beaucoup d'espèces de poissons recherchant de meilleures conditions de vie.

En ce qui concerne la répartition temporelle des espèces durant les trois années de suivi, les plus grandes richesses spécifiques sont notées en saison froide au cours de chaque année. Certes, plusieurs facteurs peuvent expliquer ce phénomène. Mais celui qui nous semble être le plus vraisemblable est la remontée des eaux froides « upwelling » qui favorise l'enrichissement en sels nutritifs et assure le développement d'un important peuplement phytoplanctonique (Dia, 1983 ; Touré, 1983).

Il convient de noter également que cette période de saison froide coïncide durant ces trois dernières années avec l'instauration du repos 
biologique pour certaines espèces de poissons et d'interdiction de la pêche nocturne au niveau de la petite côte. De telles mesures de gestion des pêcheries, en réduisant considérablement les traversées de l'AMP de JoalFadiouth vers les zones de pêches par des centaines de pirogues, favorisent la stabilité des habitats halieutiques et par conséquent le bon fonctionnement écologique de l'AMP. Cet équilibre écologique favorise le retour des espèces et leur reproduction.

\section{Conclusion}

La réalisation de cette étude qui s'est étalée sur la période 2015-2017 est sans doute l'inventaire le plus exhaustif, tant aux plan spatial que temporel, jusque-là mené sur la diversité du peuplement de poissons de l'AMP de JoalFadiouth. Elle a non seulement permis d'actualiser la liste des espèces de poissons vivant ou fréquentant l'AMP, mais aussi de connaître la répartition spatiale des différentes espèces au cours des quatre saisons hydrologiques de l'année.

Cette étude a permis d'identifier 120 espèces réparties entre 86 genres et appartenant à 48 familles. Comparés aux résultats des études antérieures sur l'AMP de Joal-Fadiouth, près de 57 espèces se sont ajoutées. Un nombre assez élevé qui incite à revoir la méthodologie et les modes opératoires lors de ces inventaires. Par contre, seules deux (02) espèces (Pisidonophis semicinctus et Aluterus punctata), qui avaient été signalées autrefois par divers auteurs, n'ont pas été retrouvées avec cette étude.

Au-delà de sa richesse spécifique assez importante, comparée aux autres AMP du Sénégal, des variations des peuplements de poissons ont été notées dans le temps et dans l'espace avec parfois de grandes fluctuations de la composition spécifique et des abondances au niveau des différentes stations et en fonction des saisons. Ces variations peuvent s'expliquer par les caractéristiques physiques des zones et des facteurs bioécologiques qui conditionnent le fonctionnement halieutique de ces zones et qui évoluent en fonction de la période de l'année. Certainement c'est pour ces raisons que la station Kher ciment, du fait de ces conditions biologiques plus favorables pour les poissons, avec sa proximité avec la zone à herbiers marins et la zone des récifs artificiels, est la station qui présente le plus grand nombre d'espèces au cours du temps. La saison froide est la période durant laquelle les plus grands nombres d'espèces sont signalés dans l'AMPJF. Une particularité fonctionnelle liée au phénomène de remontée des eaux froides « Upwelling » qui se produit durant cette période et favorisant une grande production planctonique pour l'alimentation de la faune halieutique. 


\section{References:}

1. Agardy T. (1998). Global trends in marine protected areas. In Trends and future challenges for U.S. National Ocean and coastal policy, pp. 51-55.

2. Alcala A.C., Russ G.R. (1990). A direct test of the effects of protective management on abundance and yield of tropical marine resources. ICES Journal of Marine Science 46, 40-47.

3. Allison G.W., Lubchenco J., Carr M.H. (1998). Marine reserves are necessary but not sufficient for marine conservation. Ecological Applications 8, S79-S92.

4. Attwood C.G., Harris J.M., Williams A.J. (1997). International experience of marine protected areas and their relevance to South Africa. South African Journal of Marine Science 18, 311-332.

5. Baran, E. (1995). Dynamique spatio-temporelle des peuplements de poissons estuariens en Guinée - relations avec le milieu abiotique. Thèse de doctorat en Océanologie biologique. Université de Bretagne Occidentale. 245p.

6. Beckley L.E. (1985). The fish community of East Cape tidal pools and an assesment of the nursery function of this habitat; S. Afr. J. Zool.; 2 $0 ; 2$ 1-27

7. Bell J.D. (1983). Effects of depth and marine reserve fishing restrictions on the structure of a rocky fish assemblage in the northwestern Mediterranean sea. Journal of Applied Ecology 20, 357-369.

8. Blaber S.J.M., Young J.W. and Dunning M.C. (1985). Community structure and zoogeographic affinities of the coastal fishes of the Dampier region of North-western Australia. ht. J. Freshw. Res., 36 : 247-266.

9. Blache J., Cadenat J., Stauch A. (1970). Clés de détermination des poissons de mer signalés dans l'Atlantique oriental (entre le 20ème parallèle $\mathrm{N}$ et le 15ème parallèle $\mathrm{S}$ ) ; Faune tropicale XVIK; ORSTOM ; Paris ; 479p.

10. Bretagnolle V., Duncan P., Fritz H., Lebreton J.-D. (2000). Indicateurs de l'impact des activités humaines sur l'évolution de la biodiversité. Pertinence de l'utilisation des bases de données à long terme : cas des oiseaux d'eau en zones humides. CNRS, Chize, Beauvoir sur Niort, France.

11. Caddy J.F. (2000). A fisheries management perspective on marine protected areas in the Mediterranean. Environmental Conservation 27, 98-103.

12. Cyrus D.P. and Blaber S.J.M. (1987). The influence of turbidity on juvenile marine fishes in estuaries. Part 2. Laboratory studies, 
comparison with field data and conclusions. J. Exp. Mar. Biol. Ecol., 109: 71-91.

13. DAMCP. (2014). Le suivi écologique dans l'AMP de Joal-Fadiouth (Sénégal) : Caractérisation du peuplement de poissons en 2014. Rapport réalisé avec l'appui du projet Gowamer. Dakar, décembre 2014, 16 pp.

14. Dia, A. (19S3). Observations océanographiques effectuées en 1983. Arch. Centre Rech. Océanogr. Dakar-Thiaroye, 126 : 77 p.

15. Diouf, P.S. (1996). Les peuplements de poissons des milieux estuariens de l'Afrique de l'Ouest : l'exemple de l'estuaire hyperhalin du Sine Saloum. Thèses et Documents Microfichés 156, ORSTOM, $177 \mathrm{pp}$.

16. Ecoutin, J.M., Sadio, O., Simier M., Raffray, J., Tito de Morais, L. (2012). Comparaison des peuplements de poissons d'une aire protégée en zone de mangrove (le bolon de Bamboung, Sine Saloum, Sénégal) avec les peuplements de deux sites proches non protégés de l'exploitation halieutique. Rapport $\mathrm{du}$ Contrat $\mathrm{n}^{\circ}$ CSRP/AFD/C11/2011. Dakar, juin 2012, 67 pp.

17. Francour P., Harmelin J.-G., Pollard D., Sartoretto S. (2001). A review of marine protected areas in the northwestern Mediterranean region: siting, usage, zonation and management. Aquatic Conservation: Marine and Freshwater Ecosystems 11, 155-188.

18. Freon, P., 1986. Réponses et adaptations des stocks de Cupleidés d'Afrique de l'Ouest à la variabilité du milieu et de l'exploitation. Analyse et réflexion à partir de l'exemple du Sénégal. Thèse de doctorat es sciences. Université d'Ai Marseille II. 287p.

19. García-Rubies A., Macpherson E. (1995). Substrat use and temporal pattern of recruitment in juvenile fishes of the Mediterranean littoral. Marine Biology 124, 35-42.

20. García-Rubies A., Zabala M. (1990). Effects of total fishing prohibition on the rocky fish assemblages of Medes Islands marine reserve (NW Mediterranean). Scientia Marina 54, 317-328.

21. Harmelin-Vivien M.L., Harmelin J.G., Leboulleux V. (1995). Microhabitat requirements for settlement of juvenile sparids fishes on Mediterranean rocky shores. Hydrobiologia 300, 309-320.

22. Jaccard, P. (1908). Nouvelles recherches sur la distribution florale. Bull ; soc ; vaudoise Sci. Nat ; 44 :223-270.

23. Kinzing, A. P., S. W. Pacala \& D. Tilmann (Eds.). (2002). The functional consequences of biodiversity: Empirical progress and theoretical expectations. Princeton University Press, Princeton, N. J. Kohonen, T. 1982. Self-organized formation of topologically correct feature maps. Biological Cybernatics, 43 : 59-69. 
24. Lalèyè P. (1995). Ecologie comparée de deux espèces de Chrysischthys, Poissons siluriformes (Claroteidae) du complexe lagunaire lac Nokoué - lagune de Porto-Novo au Bénin. Thèse de doctorat, Université de Liège, Liège, p.152.

25. Lenanton R.C.J. and Hodgkin E.P. (1985). Life history strategy of fish in some temperate Australian estuaries. In: Fish community ecology in estuaries and coastal lagoons : towards an ecosystem integration. Mexico, Yanez-Arancibia A. (Edit.): 267-284.

26. Lévêque C., Paugy D. \& G.G. Teugels (eds). (1990-1992).-Faune des poissons d'eaux douces et saumâtres de l'Afrique de l'Ouest. $910 \mathrm{p}$. Édit. ORSTOM.

27. Lévêque, C., Paugy D., Teugels G. (1992). Faune des poissons d'eaux douces et saurnâtres d'Afrique de l'Ouest ; MRAC/ORSTOM ; collection Faune tropicale no XXV1ll ; 2 tomes ; 902 p.

28. Livingston R.J. (1967). Field sampling in estuaries: the relationship of scale to variability; Estuaries; 10 (3); 194-207.

29. Lubchenco J., Palumbi S.R., Gaines S.D., Andelman S. (2003). Plugging a hole in the ocean: the emerging science of marine reserves. Ecological Applications 13, S3-S7.

30. Macpherson E., Biagi F., Francour P., García-Rubies A., Harmelin J.G., Harmelin-Vivien M.L., Jouvenel J.Y., Planes S., Vigliola L., Tunesi L. (1997). Mortality of juvenile fishes of the genus Diplodus in protected and unprotected areas in the western Mediterranean Sea. Marine Ecology Progress Series 160, 135-147.

31. Magnuson-Stevens Fishery Act. (2007). Management Reauthorization Act of 2006. Public Law 479.

32. Man-Waï R. (1985). Les sars du Golfe du Lion. D. sargus, D. vulgaris, D. annularis (Pisces, Sparidae). Ecobiologie - Pêche. Université des Sciences et Techniques du Languedoc-Roussillon, Perpignan.

33. Murray S.N., Ambrose R.F., Bohnsack J.A., Botsford L.W., Carr M.H., Davis G.E., Dayton P.K., Gotshall D., Gunderson D.R., Hixon M.A., Lubchenco J., Mangel M., MacCall A., McArdle D.A. (1999). No-take reserve networks: sustaining fishery populations and marine ecosystems. Fisheries 24, 11-25.

34. Pauly D., Christensen V., Guénette S., Pitcher T.J., Sumaila U.R., Walters C.J., Watson R., Zeller D. (2002). Towards sustainability in world fisheries. Nature 418, 689-695.

35. Pielou E.C. (1969). An Introduction to Mathematical Ecology. John Wiley- Interscience éd., New York, 286p.

36. Planes S., Galzin R., García-Rubies A., Goñi R., Harmelin J.-G., Le Diréach L., Lenfant P., Quetglas A. (2000). Effects of marine protected 
areas on recruitment processes with special reference to Mediterranean littoral ecosystems. Environmental Conservation 27, 126-143.

37. Planes S., Macpherson E., Biagi F., Garcia-Rubies A., Harmelin J., Harmelin-Vivien M., Jouvenel J.-Y., Tunesi L., Vigliola L., Galzin R. (1999). Spatio-temporal variability in growth of juvenile sparid fishes from the Mediterranean littoral zone. Journal of the Marine Biological Association of the United Kingdom 79, 137-143.

38. Potter, I.C., Beckley, L.E., Whitfield, A.K. and Lenanton R.C.J. (1990). Comparisons beetween the role played by estuaries in the life cycles of fishes in temperate Western Australia and Southem Africa. Environmental biology of fishies 28: 143-178.

39. Roberts C.M., Polunin N.V.C. (1993). Marine reserves: simple solutions to managing complex fisheries? Ambio 22, 363-368.

40. Rogers C.S., Beets J. (2001). Degradation of marine ecosystems and decline of fishery resources in marine protected areas in the US Virgin Islands. Environmental Conservation 28, 312-322.

41. Rossignol, H. (1973). Contribution à l'étude du complexe Guineen. ORSTOM : $142 \mathrm{p}$.

42. Roy, P. S., R. J. Williams, A. R. Jones, I. Yassini, P. J. Gibbs. B. Coastes, R. J. West, P. R. Scanes, J. P. Hudson \& S. Nichol. (2001). Structure and function of South-east Australian estuaries. Estuarine, Coastal and Shelf Science 53: 351-384.

43. Sadio, O. (2015). Evaluation de l'efficacité des Aires Marines Protégées comme outil de restauration des ressources marines et de gestion des stocks halieutiques : l'expérience ouest africaine. Thèse de doctorat en Ecologie halieutique, Université de Bretagne Occidentale, 256p.

44. Sarré A., Fall M., Ndour C. et Deme L. (2009). Caractérisation de l'état de référence de l'aire marine protégée de Joal-fadiouth, Cayar et saintLouis. Rapport d'étude. CRODT, Dakar, 58p.

45. Sène C. (2014). Potentialités et limites de la gestion participative des Aires Marines Protégées : Le cas de Joal-Fadiouth, Sénégal. Thèse de doctorat en Sciences et gestion de l'Environnement. Université de Liège, $382 \mathrm{p}$.

46. Shannon C.E. and Weaver W. (1949). The mathematical theory of communication. University Illinois Press, Urbana, 177p.

47. Sherman, K. \& E. D. Anderson. (2002). A modular approach to monitoring, assessing and managing large marine ecosystems. In J. M. McGlade, P. Cury, K. A. Koranteng \& N. J. Hardman-Mountford (Eds.), The Gulf of Guinea Large Marine Ecosystems. Op. cit.

48. Simier, M., Blanc, L., Aliaume, C., Diouf, P.S., Albaret, J.J. (2004). Spatial and temporal structure of fish assemblages in an "inverse 
estuary", the Sine Saloum system Senegal. Estuar. Coast. Shelf Sci. 59, 69-86.

49. Thiam N., Fall M., Thiaw M. (2013). Etude sur l'état des pêcheries côtières, des stocks et de leurs habitats au Sénégal. Rapport final, PRAO-SN/DPM, Dakar, Sénégal, 91p.

50. Toure, D. (1983). Contribution à l'étude de l' « upwelling » de la baie de Gorée (Dakar - Sbnégal) et de ses conséquencessur le développement de la biomasse phytoplanctonique. Dot. Sci. Centre Rech. Océanogr. Dakar-

51. Warburton K. (1978). Conununity structure, abundance and diversity of fish in a Mexican coasta1 lagoon system. Estuarine and Coastal Marine Science, 7: 497-519.

52. Weiss, M., Baret, F., Yneni, B.M., Ragnere, A.P., Nyazikhin, Y.K. (2000). Investigation of a model inversion technique to estimate canopy biophysical variables from spectral and directional reflectance data. Agronomie, EDP Sciences, 2000, 20 (1), pp.3-22.

53. White A.T., Loke C.M., De Silva M.W.R.N., Guarin F.Y. (1990). Artificial reefs for marine habitat enhancement in Southeast Asia. ICLARM Education Series 11, 45.

54. Wootton R.J. (1992). Fish ecology.- Glasgow, London, New York, Blackie Son Limited, 212 p. 